\section{LETTERS TO THE EDITORS}

The Editors do not hold themselves responsible for opinions expressed by their correspondents. No notice is taken of aronymous communications

\section{Probable Side-effect of Nuclear Reactions in the Biological Action of Fast Protons}

DRY dormant seeds of Bonus barley have been irradiated with $160-\mathrm{MeV}$. protons in the scattered beam of the Uppsala synchrocyclotron. The following biological effects were studied: (a) retardation of seedling growth under greenhouse conditions, at $20^{\circ} \mathrm{C}$.; (b) germination and (c) survival at maturity under field conditions; (d) sterility (per cent sterile flowers per spike); and (e) mutation frequency estimated from the frequency of spikes giving rise to recessive chlorophyll-aberrant types (cf. Gustafsson ${ }^{1}$ ).

From the specific ionization of the high-energy protons (see Table 1) their efficiency in producing the effects mentioned is expected to be between those of $\gamma$ - and X-rays. Bombarding animal material with fast deuterons, Tobias et al. ${ }^{2}$ found an efficiency not very different from that of X-rays. Roughly, this is in accordance with our results. A more detailed analysis indicates, however, that the fast protons are somewhat more efficient than X-rays, and further, that certain qualitative effects are intermediate between those of sparsely ionizing (X-and $\gamma$-rays) and densely ionizing radiations (pile neutrons and $\alpha$-rays).

Some of the differences between the effects of sparsely ionizing and densely ionizing radiations, which have been reviewed ${ }^{8,4}$ in order to demonstrate frequency of the remaining 'rare' types seems to increase with increasing ionization density. With due regard to the statistical uncertainty of the values quoted, it can be stated that in this respect also the proton effect is different from that expected, resem. bling that of densely ionizing radiations.

An examination of possible explanations led us to conclude that the unexpected effects must be ascribed to secondary ionization caused by shortrange particles (mostly $\alpha$-particles and low-energy protons) formed in inelastic collisions of the highenergy protons with the nuclei of the seed material. The mechanism of such nuclear reactions is not yet known in any detail, but investigations of stars in nuclear emulsion ${ }^{5,6}$ seem to indicate that roughly 10 per cent of the incident proton energy is carried away by short-range particles. Using the overall cross-section for star formation in light nuclei (carbon, nitrogen, oxygen) with $130 \mathrm{MeV}$. protons given by Lees et al. ${ }^{6}$ (cf. ref. 7), we have estimated the mean energy loss by dense ionization as about 2 per cent of the total energy dissipated. Since the relative biological efficiency of densely ionizing particles for the production of most biological effects in barley is about twenty times the efficiency of X-rays ${ }^{3,4}$, the effects of short-range particles from nuclear events will be comparable to those of the directly ionizing protons. (Measurements on the scattered proton beam have shown some contamination by fast neutrons of unknown energy spectrum, originating in inelastic collisions of the protons with the scattering target, the wall of the seed container, etc. These neutrons might

Table 1. Qcalitative Characteristics of the Effects in Barley of Monizing Radiations

\begin{tabular}{|c|c|c|c|c|c|}
\hline \multirow[b]{2}{*}{ Effect studied } & \multicolumn{5}{|c|}{ Radiation } \\
\hline & $\begin{array}{c}\text { Cobalt- } 60 \\
\gamma \text {-rays }\end{array}$ & $\begin{array}{c}160-\mathrm{MeV} \\
\text { protons }\end{array}$ & $\underset{\text { (unfiltered) }}{180-\mathrm{kV} .}$ X-rays & Pile neutrons & $\begin{array}{c}\text { Radon } \\
a \text {-rays }\end{array}$ \\
\hline $\begin{array}{l}\text { 1. Survival at maturity (per cent of seeds sown) at } 25 \text { per } \\
\text { cent germination } \\
\text { 2. Dose giving } 50 \text { per cent lethality } \\
\text { Dose giving } 50 \text { per cent sterility } \\
\text { 3. Relative frequency (per cent) of 'rare' chlorophyll } \\
\text { mutants. (In brackets, total number of mutants } \\
\text { investigated) } \\
\text { 4. Effect of water content on radiation sensitivity of seeds } \\
\text { 5. Specific ionization (ion pairs per } \mu \text { tissue) }\end{array}$ & $\begin{array}{l}20 \\
0 \cdot 92 \\
6 \cdot 6 \\
(319) \\
\text { great } \\
8\end{array}$ & $\begin{array}{l}9 \\
1 \cdot 67 \\
10 \cdot 3 \\
(639) \\
\text { small } \\
16\end{array}$ & $\begin{array}{l}17 \\
0 \cdot 95 \\
8 \cdot 6 \\
(248) \\
\text { appreciable } \\
\approx 100\end{array}$ & $\begin{array}{c}0 \\
1 \cdot 92 \\
12 \cdot 3 \\
(399) \\
\text { none* } \\
\approx 1,000-3,000 \\
\underset{\approx}{\approx} 400^{*}\end{array}$ & $\begin{array}{c}>1 \\
23 \cdot 1 \\
(65) \\
3,700\end{array}$ \\
\hline
\end{tabular}

* Cyclotron-produced neutrons from $\mathrm{Be}(d n) \mathrm{B}, 25-\mathrm{MeV}$. deuterons

a different mechanism of action of the two types of radiation, are summarized in Table 1 . In line 1 the influence of ion density on the mode of dying of plants in the field is illustrated: after treatment with $\mathrm{X}$ - and $\gamma$-rays, the seeds are killed at once or recover and give mature plants, whereas the lethal effect of neutrons is manifested later in the seedling stage, when mitoses become important for the further growth of the plant. Line 2 shows the highly sterilizing effect of densely ionizing radiations even at doses permitting considerable survival, whereas with sparsely ionizing radiations high sterility is obtained only when a large fraction of the plants is killed. In both these respects the fast-proton effect is intermediate between those of $\mathrm{X}$-rays and neutrons. The same is clearly demonstrated as regards the influence of the water content of the seeds on their sensitivity to radiation, which in the region of 7-20 per cent water increases with decreasing water content ${ }^{3}$, an effect totally absent when the irradiation is performed with neutrons.

In the mutation analysis the mutations are referred to different phenotype groups ${ }^{1}$, the albina, viridis and xantha types being most common. The relative be responsible for part of the dense ionization effect observed.) This is in accordance with the biological observations reported here. In wet tissue (for example, germinating seeds or most animal tissue) the relative importance of the effect of dense ionization will decrease, in many cases to about 50 per cent of the value obtained in dry seeds ; but it must still be taken into consideration, for example, in radiological uses of high-energy particles.

A full account of this work will appear in Hereditas. LARS EHRENBERG

Institute of Organic Chemistry and Biochemistry, University, Stockholm.

Göran ANDERSSON

Gustaf Werner Institute for Nuclear Chemistry, University, Uppsala. March 27.

${ }^{1}$ Gustafsson, Å., Lunds Univ. Årsskr., N.F. Avd. 2, 36, No. 11, 1 (1940).

'Tobias, C. A., Anger, H. O., and Lawrence, J. H., Amer. J. Roentgenol., 67, 1 (1952).

3 Ehrenberg, L., and Nybom, N., Acta Agric. Scand., 4, 396 (1954).

- Ehrenberg, L., and Saeland, E. (in the press).

${ }^{5}$ Hodgson, P. E., Phil. Mag., 44, 1113 (1953); 45, 190 (1954).

- Lees, C. F., Morrison, G. C., Muirhead, H.. and Rosser, W. G. V., ${ }^{2}$ Cassels, J. M., and Lawson, J. D., Proc. Phys. Soc., A, 67, 125 (1954). 